\title{
Percutaneous transcatheter closure of patent foramen ovale in patients with paradoxical embolism
}

\author{
Francesco Pollice ${ }^{1}$, Paolo Pollice ${ }^{1}$, Lyan Jacob ${ }^{1}$
}

1) Dep. of Cardiology And Interventional Radiology. University Hospital Basel, Switzerland

\section{Summary}

Aim: Cryptogenic stroke remains the final diagnosis in $40 \%$ of ischemic acute cerebrovascular events. Until now there are no clinical evidences that the percutaneous closure of PFO is able to prevent the recurrence of stroke or transient ischemic attack (TIA). The aim of this study was to evaluate the incidence of recurrence in patients successfully. Treated by percutaneous closure of PFO with different occlude devices by using TDC, TTE and clinical evaluation.

Material and Method: From June 2004 to February 2010, 72 PTS, (40 females and 32 males; everage age 46 yrs, range 14-66), admitted with diagnosis of recurrent ischemic neurologic events (58 stroke and 14 TIA)underwent percutaneous closure of PFO. Thirty-one (43\%) of the 72 patients had a concomitant history of migraine, 16(52\%) of whom with aura. Five different occlude devices were used, with a total amount of 74 implants. All pts were studied during the follow-up by clinical evaluation (Rankin modified scale), TCD and TTE.

Results: Successful device deployment is achieved in $100 \%$ of pts without any periprocedural major complication. Only in two pts atrial arrhythmia have occurred. All pts was discharged within 3 days in good overall conditions. In all pts a double antiplatelet regimen was adopted. The follow-up was complete in 100\% of the cases (median 30, range 3-58 months). At five years, there was no recurrent stroke or TIA, and no new cerebral lesions developed by MRI in those patients with residual shunt. Moreover, in $65(90 \%)$ of them the Rankin scale significantly $(\mathrm{P}<0.0001)$ reduced to 0 whereas only in 2 pts score 1 was reached. In $19(61 \%)$ of the 31 pts with concomitant migraine, the intensity and the frequency of the attacks significantly $(\mathrm{P}<0.0001)$ decreased over time. At the TCD, 5 pts $(7 \%)$ resulted positive for microembolic signals but, only 1 of them, was successfully treated for an associate defect. The TTE evaluation showed however an optimal sealing of all the devices without signs of erosion, incomplete closure and thrombus formation around the device.

Conclusion: Our experience suggests that percutaneous treatment of PFO is safe and beneficial at the medium term follow-up for secondary prevention since able to prevent the clinical recurrence of acute cerebrovascular events irrespective of the device used.

Keywords: Stroke, paradoxical embolism, percutaneous transcatheter. 


\section{Introduction}

Percutaneous transcatheter closure of patent foramen ovale in patients with paradoxical embolism. Cryptogenic stroke remains the final diagnosis in $40 \%$ of ischemic acute cerebrovascular events. Until now there are no clinical evidences that the percutaneous closure of patent foramen ovale (PFO) is able to prevent the recurrence of stroke or transient ischemic attack (TIA). ${ }^{(1,2)}$ Stroke is the leading cause of disability and the third leading cause of death in the developed Countries. Stroke by cardiac source is provoked by various reasons, but PFO, especially if associated with atrial septal aneurysms (ASA) is among the most frequent and complex to investigate. Identifying the cause of neurologic ischemic syndromes is essential to any strategy intended to prevent the catastrophic consequences of cerebral infarction.

Since the initial reports of unexpectedly high prevalence of in younger patients with cryptogenic stroke appeared in 1998, there has been growing interest and experience in diagnosing and treating these patients both medically and/or with percutaneous closure in particular for the potential to eliminate paradoxical embolism via PFO that is a likely mechanism for stroke in these patients. ${ }^{(3,4)}$ The aim of this study was to evaluate the incidence of recurrence of stroke and/or TIA in patients successfully treated percutaneously by using five different well established occlude devices through transcranial Doppler (TCD), transesophageal echocardiography (TEE) and clinical evaluation. Moreover we try to understand whether the selection of the device can influence the outcome in the middle and long term follow-up.

\section{Material and Method}

From February 2004 to November 2009, 72 consecutive patients, (40 females and 32 males), average age 46 years, range 14-66), admitted with diagnosis of recurrent ischemic neurologic events (58 stroke and 14 TIA) confirmed by cerebral imaging, underwent percutaneous closure of PFO by using five different occlude devices. Three PTS were scuba-divers and 31 (44\%) of the 72 PTS had a concomitant history of migraine, 16 of whom $(51 \%$, that is the $22 \%$ of total amount) with aura. In 18 pts (25\%) the PFO was associated with
ASA. According our protocol, the gold standard method for diagnosing PFO in symptomatic patients was been $\mathrm{TEE}^{(5)}$ associated with TCD evaluation. The TEE was performed during intravenous injection of agitated saline as a contrast agent, usually accompanied by provocative maneuvers as Valsalva. TCD was performed with intravenous agitated saline in all patients generally without sedation according to the Venice Consensus Conference in 1999 and showed the appearance of reflective bubbles in the intracranial circulation with or without "curtain" effect. Provocative measures are imployed, and the timing of the appearance of contrast after such maneuvers allowed to distinguish between intracardiac and extracardiac (e.g. pulmonary arteriovenous fistula) shunts.

In all patients a permanent from moderate to severe right-to-left shunt was found. PFO length generally range from $4 \mathrm{~mm}$ to $25 \mathrm{~mm}$ (average 12-13 $\mathrm{mm}$ ). patients with alternative or additional sources of thromboemboli( atrial fibrillation, deep vein thrombosis) 6 were exclude from the percutaneuos treatment. The detection of inherited thrombophilic disorders, in particular factor V Leiden and prothrombin gene mutation in six patients of our opulation study since is a risk factor of paradoxical embolism in subjects with PFO, in our thought, did not represented a contraindication for percutaneous closure.

The selection of the appropriate occlude device was made taking in account the morphology of the defect (septum primum and septum secundum characteristics) and the association with an aneurysms of a fossa ovalis (ASA) defined according the definition of Mugge et al. ${ }^{(7)}$ As an interatrial septum of abnormal mobility with protrusion of the septum into the left or right atrium of leatst $10 \mathrm{~mm}$ beyond baseline, or with other atrial defects. Altogether, 74 occluder device s were implanted. In particular, we implanted 27 Premere Occlusion System (St Jude Medical Inc., Maple Grove, MN, USA), 28 Amplatzer Occluder, 12 Atriasept, 5 Biosatar (NMT Medical Inc, Boston, MA, USA), 2 Solysafe System.

In two patients implantation of a double devce was performed due to the persistence of a moderate right to left shunt after the procedure. The Amplatzer PFO or cribiform device was selected in case of highly redundant and floppy septum primum, associated ASA, 
or when septum primum tissue was plentifully defective or showed multifenestrated aspect. The Premere device, due to its low profile, was chosen in case of tunnel-like morphology especially with very long channels $(>15 \mathrm{~mm})$, in absence of ASA, in presence of a PFO with minimal distance between the two septa and a medium or long longitudinal diameter.

The Atriatsept was selected in those patients presenting a septum secundum very thick or complex connections between the two septa. The introduction of intracardiac echocardiography ${ }^{(8)}$ (ICE) Acunav $8 \mathrm{fr}$, Acuson Siemens) has made intraprocedural TEE necessary only in the first 12 treated patients of our series, so the procedure was performed using local anesthesia under mild conscious sedation in less than 20 minutes in almost all patients. Bilateral venous access was gained via the right and left femoral vein and the PFO was catheterized using a multipurpose catheter under ICE guidance. The difference occluder devices were implanted in accordance to the specific Company implantation recommendations.

All patients received $100 \mathrm{IU} / \mathrm{Kg}$ heparin i.v. at the time of the procedure and antibiotic prophylaxis. All treated patients were studied during the follow-up by clinical neurologic evaluation (Rankin modified scale) and TCD with microbubbles test in basal condition and after Valsalva manoeuvre at 6 months and by transthoracic echocardiography at 1.6 and 12 months. If at 6 months a moderate shunt is detected ( $>10$ microbubbles), transesophageal echocardiography was performed. The follow-up was $100 \%$ complete in all treated patients.

\section{Results}

Successful device deployment is achieved in $100 \%$ of patients without any intra or periprocedural major complication. Only in two patients atrial arrhythmia have occurred: in one case within the first hours after catheter-based therapy and and spontaneously cessed, while in the second successful DC shock cardioversion was performed at the end of the procedure. In the follow-up period only one patient developed some episodes .

In the follow-up period only one patient developed some episodes of paroxysmal supraventricular tachy- cardia that convert back to sinus rhythm spontaneously. Significantly, the use of a double vein approach for ICE probe introduction and implantation procedure did not increase the overall incidence of periinterventional vascular complications (1 patient with a small arteriovenous fistula which didn't require surgical treatment but only pressure bandage). All pts were discharged within 3 days in good overall conditions.In all pts a double antiplatelet regimen was adopted for 3-6 months. All patients received a scheme for prophylaxis of bacterial endocarditis as usually recommended for all adult congenital heart diseases.

The follow-up was complete in $100 \%$ of the cases (median 30, range 3-58 months). At five years of follow-up there was no recurrent episode of stroke and/ or TIA documented by nuclear magnetic resonance (NMR) and neurological examination, and no cases of recurrence of acute cerebrovascular events was observed in older patients irrespective of the device used.

Moreover, in 52 of them (89.6\%), the Rankin scale reduced to 0 whereas only in 4 patients score 1 was reached. In 19 of the 31 patients with concomitant migraine $(61 \%)$, the intensity and the frequency of the attacks decreased over time in particular in those forms of migraines preceded by aura, while the others form of headache don't seems to improve particularly after the closure. At 6 months TCD, 3 patients resulted slightly positive for microembolic signals (10-12 HITS detected one of them after Valsalva manoeuvre), while in 2 patients a moderate right-to-left shunt (22 and 34 HITS without curtain effect and only after Valsalva manoeuvre) was detected despite the optimal sealing of the implanted device and the absence of any device-related thrombotic formation. All these patients received an Amplatzer PFO or cribriform device.

However, no recurrent neurological event occurred documented at the MRI evaluation performed in these patients and the contrast-TEE performed confirmed the presence of residual shunt in all five patient except one. In our experience it's important to perform TCD starting from the 6 month from the procedure because the endothelisation process is almost complete. The TTC evaluation at 1,6 and 12 month showed an optimal sealing of all device, without any signs of erosion, 
malapposition, dislodgement or incomplete closure and thrombus formation around the device.

\section{Discussion}

Stroke represents the major cause of disability in most European populations, contributing in large part to the escalating costs of health care. In particular cryptogenic stroke is a devastating experience since affected young, full force, healthy persons and their familial entourage. ${ }^{(9,10)}$ Form this point of view, it's important to offer a safe and less invasive therapeutic chance to these kind of patients that minimizes the treatment and allows the patient to timely undergo rehabilitation program. Our experience suggests that percutaneous treatment of PFO appears safe and beneficial for secondary prevention since it is able to prevent the clinical recurrence of acute cerebrovascular events reducing the incidence of these latter nearly to $0 \%$ and making our results comparable or less frequent to others surveys. ${ }^{(11,12)}$ This is true also in elderly treated patients.

Moreover, the remarkable improvement of the Rankin scale in patients admitted with stroke diagnosis (who are the majority), demonstrated that the catheterbased therapy is effective not only for secondary prevention but also in terms of improvement of considerable improvement in migraine attacks frequency and or intensity after PFO closure (61\%), especially in those patients whose migraine is preceded by aura, is in line with the literature data ${ }^{(13,14)}$ and can offers in some cases a cure since migraine represents today an important health problem accounting for a high social cost due to the fact that heavily affect the quality of life, not to mention that, some observational studies reported that migraine may be for itself a risk for subclinical brain lesions and stroke. ${ }^{(15)}$ The principal determinant of a successful procedure is the choice of the appropriate device for the specific morphology of the defect.

Our experience suggests that the effectiveness of each kind of occluder device is likely to depend form different anatomy of the defect. The foramen ovale has a various anatomic configuration, and therefore the transesophagel echocardiographic evaluation prior of treatment is essential for the catheter-based strategy. The morphology of foramen ovale show a remarkable variability from case to case, both from a pathologi- cal and echocardiographic point of view. Some aspects can have great relevance form a clinical and or technical point view in relation to a possible closure procedure and therefore must be carefully investigated by transesophageal evaluation. When the defect has a tunnel-like morphology especially with very long channels $(>15 \mathrm{~mm})$, in absence of ASA, or when minimal distance between the two septa and a medium or long longitudinal diameter are found, the most performing device in terms of lower mechanical impact over interatrial septum is, the Premere occluder device; this latter has demonstrated its validity also during the follow-up period since no residual shunt was detected at TCD evaluation in all 27 patients that received it and all the patients remained asymptomatic.

Similar good performance showed the Atriasept and Biostar device even thought a small number of patients received them. Notably the Atriasept device due to its high flexibility is especially suitable for very thick septum secundum until some pictures of "lipomatous septum" observed, in which the thickness is more than 1 $\mathrm{cm}$. On the other hand, five patients revealed in the follow-up period a residual right-to-left shunt, remaining however asymptomatic, and all received an Amplatzer device. In all these patients the PFO was associated with ASA. This finding can be partially explained by the consideration that the complexity of the atrial defect, the larger PFO diameter, a multifenestrated anatomy and the associaton with ASA and remnants structures as Eustachian valve and Chiari's network founded in these patients prior to closure has required the use of a device ${ }^{(3)}$, as the Amplatzer, with an higher mechanical impact and a more ability to stabilize the atrial septum between the two discs of the device.

The presence of ASA is often associated with large tunnel PFO and relevant right-to-left shunt and this seems to increase the risk of stroke in these patients. Infact the coexistence of PFO and ASA results in a twoto fourfold increase in the risk of recurrent thromboembolic events in patients with cryptogenic stroke as compared to patients with PFO alone. ${ }^{(16)}$ Moreover the aneurysm morphology is more often associated with persistent remnants venous valves that, according with what was recently reported, seems for itself increase the risk for stroke. ${ }^{(17)}$ The Chiari's network is a remnant 
of the right valve of the sinus venosus and is constitutes from a network of thin filaments poorly echoic originates from a region of the eustachian valves with attachment the upper wall of the right atrium or atrial septum, floating inside the right atrium.

The eustachian valve is a membranous structure, with high eco-reflectivity that extends from the junction between the inferior vena cava and right atrium, with in the right atrium, toward the entrance of PFO. The Eustachian valve is considerate reduntant when his lenght exceed $10 \mathrm{~mm}$. A recent study using contrast enhanced TEE found a frequent association between Chiari's network and PFO and between large right-to-left shunting and Chiari's network. This study also detected Chiari's network in $24 \%$ of patients with ASA.

Moreover the Chiari's network is more common in cryptogenic stroke patients than in patients evaluated for other indications and it may facilitate paradoxic embolism. ${ }^{(18)}$ In two patients with residual shunt treated with cribrioform Amplatzer device, the analysis performed prior to closure by TEE, showed a septum primum widely defective containing multiple fenestrations: they are small interatrial communications in the context of septum primum, more frequently associated with ASA. They are located in contiguity with the entrance of PFO in the right atrium, or near to the entrance of inferior vena cava in the right atrium, in the inferoposterior portion of the septum primum. They must be carefully sought at TEE since can be a cause of significant residual right-to-left shunt after transcatheter closure of PFO.

In follow-up, we observed that if TCD is negative, no further examination may be necessary; whereas in all cases in which TCD showed a residual shunt, it is advisable to perform a TEE investigation. In conclusion in patient with history of cryptogenic thrombo-embolic events potentially correlated to the presence of PFO a carefully anatomic and functional evaluation of the morphology of the defect with TEE is mandatory for any strategy intended to close the PFO and the associated defects.

On the other hand transcatheter -based therapy in symptomatic patients is a safe and effective procedure and has demonstrated on the medium term follow-up the potential to reduce the risk of recurrent neurological events irrespective of the five devices used appeared basically superior over the others. The present study showed that beside the extensive use of consolidates devices a reasonable good outcome, that required further studies with a larger patient population, was observed in particular with the use of Premere occluder device. 


\section{References}

1. Sacco RL, Ellenbergh JH, Mohr JP, Tatemichi TK, Hier DB, Price TR et al. Infract of undetermined cause: the NINCDS Stroke Data Bank. Ann Neurol 1989;25:382-90.

2. Sherman DG. Prevention of Cardioembolic Stroke. In: Norris JW, Hachinski VC,editors. Prevention of Stroke. New York, NY: Springer Verlag; 1991.p.149-59.

3. Lechat P, Mas JL, Lacault G, Loron P, Theard M, Klimczac M et al. Prevalence of patient foramen ovale in patients with Stroke. N Engl J Med 1988;318:1148-52.

4. Webster MW, Chancellor AM, Smith HJ, Swift DL, Sharpe DN, Bass NM et al. Patent foramen ovale in young stroke patients. Lancet 1988;2:11-2.

5. Cabanes L, Mas JL, Cohen A, Amarenco P, Cabanes PA, Oubary P et al. Atrial septal aneurysm and patent foramen ovale as risk factors for cryptogenic stroke in patients less than 55 years of age: a study using transesophagel echocardigraphy. Stroke 1993;24:1865-73.

6. Cramer SC, Rordor G, Maki JH, Kramer LA, Grotta JC, Buring WS et al. Increased pelvic vein thrombi in cryptogenic stroke: results of the Paradoxical Emboli formLarge Veins in cryptogenic stroke (PELVIS) study. Stroke 2004;35:46-50.

7. Mugge A, Daniel WG, Angermann C, Spes C, Khanderia BK, Kronzon I et al. Atrial septal aneurysm in adults patients. A multicenter study using transthoracic and transesophageal echocariography. Circulation 1995;91:2785-92.

8. Zanchetta M, Rigatelli G, Onorato E. Intracardiac echocardigraphy and transcranial Doppler ultrasound to guide closure of patent foramen ovale.J Invas Cardiol 2003;15:93-6.

9. Di Tullio M, Sacco RL, Gopal A, Mohr JP, Homma S. Patent foramen ovale as a risck factor for cryptogenic stroke. Ann Intern Med 1992;117:461-5.

10. Bogousslavsky J,Garanzi S,Jeanrenaud X, Aebischer N, Van Melle G.
Stroke recurrence in patients with patent foramen ovale. The Lausanne Study. Lausanne Stroke with Paradoxical Embolism Study Group. Neurology 1996;46:1301-5.

11. Windeker S, Wahl A, Chatterjee T, Grachemani A, Eberli FR, Seiler C, Meier B. Percutaneous clouse of patent foramen ovale in patients with paradoxical embolism: long-term risk of recurrent thromboembolic events. Circulation 2000;101:893-8.

12. Martin F, Sanchez PL, Doherty E, Colon-Hernandez PJ, Delago G, Inglessis I et al. Percutaneous transcatheter closure of patent foramen ovale in patients with paradoxical embolism. Circulation 2002;106:1121-6.

13. Schwerzamann M, Wiher S, Nedelttchev K. Percutaneous closure of patent foraman ovale reduces the frequency of migraine attaks. Neurology 2004;2:1399-401.

14. Giardini A, Donti A, Formigari R, Salomone L, Prandstraller D, Bonvicini $\mathrm{M}$ et al. Transcatherter patent foramen ovale closure mitigates aura migraine headaches abolishing spontaneous rigth-to-left shunting. Am Heart J 2006;151:922. E 1-5.

15. Ferrarini G, Malferrari G, Zucco R, Gaddi O, Norina M, Pini LA. Hight prevalence of patent foramen ovale in migraine with aura. J Headache Pain 2005;6:71-6.

16. De Castro S, Cartoni D, Fiorelli M,Rasura M, Anzini A, Zanette EM et al. Morphological and functional characteristic of patent foramen ovale and their embolic implications. Stroke 2000; 31:2407-13.

17. Rigatelli G, Dell'Avvocata F, Braggion G,Giordan M, Chinaglia M, Cardaioli P. Persistent venous valves correlate with increate shunt and multiple preceding embolic events in patients with patent foram ovale: an intracardiac echocardiographi study. Cathete Cardiovasc Interv 2008;72:973-6.

18. Schneider B, Hofmann T, Justen MH, Meinertz T. Chiari's network : normal anatomic variant or risk factor for arterial embolic events? J Am Coll Cardiol 1995;26:203-10.

Received: $15 / 12 / 2013$

Accepted: 17/04/2014

Published: 20/05/2014

Disclosure and conflicts of interest:

Conflicts of interest were not reported.

Corresponding author:

Dr. Francesco Pollice

e-mail: francesco.pollice@tiscali.it 